Anaesthesist 2012 - 61:5

DOI 10.1007/s00101-012-1984-6

Online publiziert: 26. Januar 2012

(c) Springer-Verlag 2012
H.J. Bardenheuer

Zentrum für Schmerztherapie und Palliativmedizin, Klinik für Anaesthesiologie, Universität Heidelberg

\section{Ethische Fallberatung in der Intensivmedizin}

Gottes Auftrag „Macht euch die Erde untertan“ (Genesis 1,28) entsprechend hat sich der Mensch zum Problemlöser entwickelt. Dabei hat er enorme Fähigkeiten und Fantasien entwickelt, die schwierigsten Probleme des Lebens zu meistern. In der Medizin sind dadurch erhebliche Fortschritte in der Behandlung von lebensbedrohlichen Erkrankungen erreicht worden, beispielsweise durch Organtransplantationen bei chronischem Organversagen, Chemotherapien bei bösartigen Krankheiten und den Einsatz aufwändiger technischer Verfahren in der Intensivmedizin.

Der Glaube an die unbegrenzten Möglichkeiten der technisierten Intensivmedizin verleitet dazu, der aktiven Auseinandersetzung mit dem Lebensende aus dem Weg zu gehen. Während wir die Therapie des progredienten Organversagens $\mathrm{zu}$ den wesentlichen Aufgaben des Arztes zählen, weisen wir die schwierige Auseinandersetzung mit dem sterbenden $\mathrm{Pa}$ tienten häufig anderen Professionen wie beispielsweise den Seelsorgern zu. Obwohl die Themen Sterben und Tod natürlicherweise alle Bereiche der Medizin betreffen, kumuliert die spezifische Auseinandersetzung beim Intensiv- und Palliativpatienten. In besonderem Maß hat die Palliativmedizin das Bewusstsein zu Fragen des Menschenbilds geschärft und die ethische Auseinandersetzung mit Entscheidungen am Ende des Lebens gefördert. Der allgemeine Umgang mit Gesundheit und Krankheit sowie die gesell- schaftliche Diskussion zu Fragen der Patientenautonomie haben die Medizinethik aus der theoretisch-philosophischen Positionierung herausgeführt und direkt am Krankenbett an der Seite des Arztes und der Pflegekräfte mit den lebensbestimmenden Fragen des kritisch kranken Patienten konfrontiert.

Der Leitthemenbeitrag dieser Ausgabe von Der Anaesthesist „Ethische Fallberatung in der Intensivmedizin" von Thomas Bein und Bernhard M. Graf zeigt auf, dass nicht nur der Patient und die Angehörigen intensive sowie kompetente ärztliche Begleitung bei der Entscheidungsfindung zur Therapiebegrenzung benötigen, sondern dass auch das auf der Intensivstation tätige Personal essenziell in die Therapie eines kritisch kranken Patienten und in die das Leben entscheidenden Prozesse einbezogen werden muss. Dabei bietet die „ethische Fallberatung“ eine Option, im konkreten Fall einen strukturierten Handlungsablauf zur ethischen Entscheidungsfindung zu gewährleisten. Die Ethikberatung hat zum Ziel, die Entscheidungsprobleme zu analysieren und potenzielle Handlungsoptionen zu erarbeiten. Die Strukturierung des Vorgehens ist ein Garant für die Objektivierbarkeit des ethisch fundierten Entscheidungsprozesses. Fatal wäre es, wenn Einzelpersonen kraft ihres Amtes bzw. ihrer persönlichen Beziehung zum Patienten oder sogar ganze Berufsgruppen das ethisch medizinische Handeln in ihrem jeweiligen Sinne beeinflussen. Fachkompetente Außen- stehende sind in der Lage, die Objektivierbarkeit der Entscheidungen durch kritischen Außenblick auf die an der Therapie beteiligten Personen zu gewährleisten.

Die Institution der ethischen Fallberatung ist heute schon an vielen Kliniken eingeführt. In dem Bewusstsein und mit dem Ziel einer menschenwürdigen $\mathrm{Me}$ dizin gehört die ethische Beratung zum Standard allen ärztlichen Handelns, insbesondere in kritischen Arbeitsbereichen wie Palliativ- und Intensivmedizin.

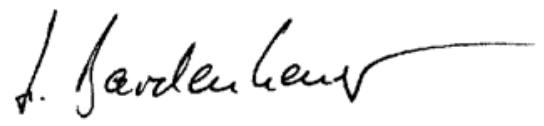

H.J. Bardenheuer

\section{Korrespondenzadresse}

Prof. Dr. H.J. Bardenheuer

Zentrum für Schmerztherapie und Palliativmedizin, Klinik für Anaesthesiologie, Universität Heidelberg

Im Neuenheimer Feld 131, 69120 Heidelberg hubert.bardenheuer@med.uni-heidelberg.de 